\title{
Equitable allocation of COVID-19 vaccines in the United States
}

\author{
Harald Schmidt ${ }^{1,9 凶}$, Rebecca Weintraub ${ }^{2,9}$, Michelle A. Williams $^{3}$, Kate Miller ${ }^{2}$, Alison Buttenheim ${ }^{4}$, \\ Emily Sadecki ${ }^{5}{ }^{5}$, Helen Wu ${ }^{6}$, Aditi Doiphode ${ }^{6}$, Neha Nagpal ${ }^{6}$, Lawrence O. Gostin ${ }^{7,0}$ and \\ Angela A. Shen 8,10
}

\begin{abstract}
Many vaccine rationing guidelines urge planners to recognize, and ideally reduce, inequities. In the United States, allocation frameworks are determined by each of the Centers for Disease Control and Prevention's 64 jurisdictions ( 50 states, the District of Columbia, five cities and eight territories). In this study, we analyzed vaccine allocation plans published by 8 November 2020, tracking updates through to 30 March 2021. We evaluated whether jurisdictions adopted proposals to reduce inequity using disadvantage indices and related place-based measures. By 30 March 2021, 14 jurisdictions had prioritized specific zip codes in combination with metrics such as COVID-19 incidence, and 37 jurisdictions (including 34 states) had adopted disadvantage indices, compared to 19 jurisdictions in November 2020. Uptake of indices doubled from 7 to 14 among the jurisdictions with the largest shares of disadvantaged communities. Five applications were distinguished: (1) prioritizing disadvantaged groups through increased shares of vaccines or vaccination appointments; (2) defining priority groups or areas; (3) tailoring outreach and communication; (4) planning the location of dispensing sites; and (5) monitoring receipt. To ensure that equity features centrally in allocation plans, policymakers at the federal, state and local levels should universalize the uptake of disadvantage indices and related place-based measures.
\end{abstract}

T he US Food and Drug Administration first granted emergency use authorizations for vaccines against coronavirus disease 2019 (COVID-19) on 8 December 2020. On 19 April 2021, all adult US residents became eligible to receive a COVID-19 vaccine; at this point, one quarter (87 million) of the US population was fully vaccinated; 46 million people required a second shot; $70-100$ million adults who would need to be vaccinated to achieve herd immunity remained unvaccinated; and around 3 million daily doses were administered ${ }^{1}$. Scarcity due to production capacity limitations and logistical constraints had become less intense than in previous months, but equitable allocation remained critical. Allocations are determined by the Centers for Disease Control and Prevention's (CDC) 64 immunization grantees (50 states, the District of Columbia (DC), five large cities and eight territoriesreferred to, collectively, as jurisdictions). The CDC requested that all jurisdictions provide allocation plans by 31 October 2020 (ref. $\left.{ }^{2}\right)$. To assist planners throughout the allocation process, we analyzed these plans and subsequent updates to understand to what extent they reflect a novel proposal by the National Academies of Science, Engineering and Medicine (NASEM) to promote equity not only across, but also within, phases of vaccine distribution. A summary of the main findings and limitations of the study is provided in Table 1.

COVID-19 vaccine allocation relates to two main processes: providing available doses to jurisdictions according to their population or other metrics ${ }^{3}$ and then, within jurisdictions, distributing vaccines among specific populations. Allocation frameworks seek to integrate a multitude of factors. Typically, they center on a risk-based approach that aims to reduce mortality and limit the spread of infections. A central question is to what extent ongoing implementation efforts align with, or stand in conflict with, commitments to mitigate inequities, particularly those affecting economically disadvantaged racial and ethnic groups who have experienced, and continue to experience, disproportionate effects of COVID-19 (refs. ${ }^{4-9}$ ).

The CDC's Advisory Committee on Immunization Practice (ACIP) traditionally provides guidance to jurisdictions in the case of pandemics. ACIP's overarching ethical framework for allocating COVID-19 vaccines notes that allocation strategies 'should aim to both reduce existing disparities and avoid creating new disparities' $^{10}$, echoing an earlier articulation of the committee's scientific and ethical principles ${ }^{11}$. A similar emphasis is found in initial proposals in the academic literature ${ }^{12}$; in influential high-level policy advice by NASEM ${ }^{4}$, which is tasked by the CDC and the National Institutes of Health with assisting ACIP in developing an equitable allocation framework; as well as in the World Health Organization's (WHO) Strategic Advisory Group of Experts on immunization $^{13}$. In this study, our concept of equity is directly aligned with NASEM's articulation. That is, we understand it as integrating both horizontal equity (requiring treating the same those who have the same needs) ${ }^{14}$ and, importantly, vertical equity (requiring treating differently those with different needs). Specifically, as NASEM exemplifies, this requires prioritizing communities that have been

'Department of Medical Ethics and Health Policy, Center for Health Incentives and Behavioral Economics, Leonard Davis Institute of Health Economics, University of Pennsylvania, Philadelphia PA, USA. ${ }^{2}$ Ariadne Labs, Brigham and Women's Hospital \& Harvard T.H. Chan School of Public Health, Boston, MA, USA. ${ }^{3}$ Harvard T.H. Chan School of Public Health, Harvard University, Boston, MA, USA. ${ }^{4}$ Department of Family and Community Health, School of Nursing; Center for Health Incentives and Behavioral Economics; Leonard Davis Institute of Health Economics, University of Pennsylvania, Philadelphia PA, USA. ${ }^{5}$ Department of Medical Ethics and Health Policy, University of Pennsylvania, Philadelphia PA, USA. ${ }^{6}$ School of Arts and Sciences, University of Pennsylvania, Philadelphia PA, USA. 'O'Neill Institute for National \& Global Health Law, Georgetown University, Washington, DC, USA. ${ }^{8}$ Vaccine Education Center, Children's Hospital of Philadelphia, Philadelphia PA, USA. ${ }^{9}$ These authors contributed equally: Harald Schmidt, Rebecca Weintraub. ${ }^{10}$ These authors

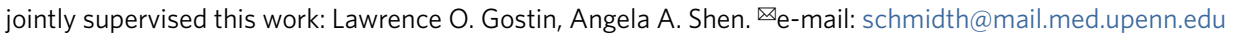




\section{Table 1 | Policy summary}

Background

Policy implications
COVID-19 disproportionately impacts disadvantaged communities. Major guidelines recommend that vaccine allocation frameworks reduce inequities. One way of improving equity is through the sequence of priority groups. A novel further option is to prioritize within each priority group, using place-based disadvantage indices throughout all phases (that is, including the general population). We ascertained the extent to which disadvantage indices and related measures were adopted in the United Staes between 8 November 2020, and 30 March 2021.

Six months after first being asked to publish their formal allocation frameworks, most states had adopted disadvantage indices or zip code-based measures to reduce inequities, for five different purposes. Although guidelines evolved rapidly, and although full formal allocation plans were not available for all CDC jurisdictions, this analysis comprises different official sources for all jurisdictions. The analysis cannot settle the exact extent to which equity or epidemiological considerations drive adoption of place-based measures of disadvantage, but at least 22 states echo expressly social justice rationales identified as foundational in previous guidance.

In a major shift in rationing frameworks, most US states have recognized the need to promote equity within allocation phases through the use of place-based disadvantage indices and related measures. Broader adoption should be promoted further, because the need for equitable allocation continues even when the entire population is eligible for vaccines. Other countries-particularly those with similarly pronounced patterns of disparities in health, wealth and COVID-19 impact-should consider which elements can still be adopted, to allocate vaccines in ways that simultaneously promote equity and public health. disproportionately impacted by COVID-19 due to persistent structural and systemic disadvantage and racism that contribute to avoidable shortfalls in health outcomes more generally ${ }^{4,12,15}$.

One way equity can be addressed is through the sequence of priority groups, which often simultaneously reflect a desire to prevent harms (such as death, hospitalization and infection) and promote equity (as risks are not distributed equally across income and racial or ethnic groups). Figure 1 shows ACIP's and NASEM's respective frameworks, depicting similarities and differences. Both frameworks prioritize healthcare workers equally; however, people with comorbidities are prioritized differently. Because structural racism worsens health and economic opportunity, these groups comprise larger shares of economically disadvantaged minorities than the general population ${ }^{5,16-20}$. NASEM places incarcerated and homeless populations before children and non-frontline critical risk workers, whereas ACIP does not address these groups specifically, instead grouping them with the general population.

Importantly, NASEM also recommended the use of an additional measure to promote equity. Within "each population group, vaccine access should be prioritized for geographic areas identified through CDC's Social Vulnerability Index (SVI) or another more specific index." ${ }^{2}$ An index such as SVI is a statistical measure tied to a geographic area that captures the relative average advantage and disadvantage of people living there, by integrating relevant metrics such as income, educational attainment and housing quality ${ }^{21,22}$. Such indices can capture population groups for which the protection offered by vaccines is both more necessary and more valuable, as they are typically more dependent on regular income, less able to socially distance and more likely to contract and spread the infection $^{6,7}$. In addition to increasing the epidemiological benefit of vaccines, disadvantage indices also address health inequities, which ACIP, NASEM and others recognize as important ${ }^{4,8,12,19}$. NASEM notes that an index such as SVI incorporates the variables that are most linked to the disproportionate impact of COVID-19 on people of color and other vulnerable populations ${ }^{4}$. To address this disparate impact, NASEM recommends setting aside $10 \%$ of federally available vaccines for vulnerable communities (as determined by SVI), to be added to the allocations that would otherwise be offered proportionately by population $\operatorname{size}^{23}$. Jurisdictions should, furthermore, make special efforts to deliver vaccines to residents in high-SVI areas (conceptualized as the $25 \%$ most disadvantaged) ${ }^{4}$. CDC staffers noted that the SVI could be integrated into Tiberius, a newly developed software. Developed by a private company, the platform is intended to assist jurisdictions with determining the shares of vaccines to be sent to particular areas within their boundaries and to track administration ${ }^{21}$. SVI-weights can be added to the allocation formulas such that more disadvantaged areas receive larger amounts. In response to data demonstrating disproportionately lower vaccine receipt by minorities after the first month of the rollout, the CDC also expressly recognized the index's utility for monitoring coverage rates and for identifying communities where focused efforts might be required to reduce inequity ${ }^{24}$. Similarly, the Biden-Harris administration's national COVID-19 strategy recommended that jurisdictions should use the SVI or other indices to describe how they have or will provide equitable vaccine access ${ }^{25}$. Public health planning aside, assessing coverage rates by, for example, SVI deciles can also support disparate impact monitoring, a legal concept focused on determining whether policies negatively affect a protected group, even if they do not have that express intention and do not directly use information about that group ${ }^{26-28}$. To ascertain the extent to which allocation guidance incorporates disadvantage indices and related measures, we, therefore, analyzed jurisdictions' plans.

\section{Results}

The initial search (7-14 November 2020) yielded a total of 63 summaries (98.4\% of all jurisdictions) and 47 full guidance documents $(73.4 \% \text { of all jurisdictions, including all states })^{29}$. Subsequent searches (8-14 December 2020; 21 December 2020-1 January 2021; 7-19 January 2021; and 20-30 March 2021) yielded one additional summary (total $n=64,100 \%$ ) and five additional full guidance documents (total $n=52,81.3 \%$ ). Twenty-four jurisdictions had one or more updates to full guidance documents, and, for 35, one or more supplemental documents were identified; the current review includes data for all 64 jurisdictions. Tables 2 and 3 and Figs. 2 and 3 summarize the findings from all searches. Because NASEM recommended the use of a disadvantage index, and to reduce complexity in general, we focused on indices but provide data on zip code use for further context.

A total of 37 jurisdictions report using a disadvantage index in different ways, applying to around 269 million residents (82\%; Fig. 3). Twenty-nine jurisdictions (28 states and one city) refer specifically to the SVI. Eleven states and two cities refer to seven other established or internally developed indices: Community Vulnerability Index (CCVI, $n=5$ ), Area Deprivation Index (ADI, $n=2$ ), and five newly developed indices (Healthy Places Index, Pandemic Influenza Vulnerability Index, and three indices all called the 'COVID Vulnerability Index', but differing from one 


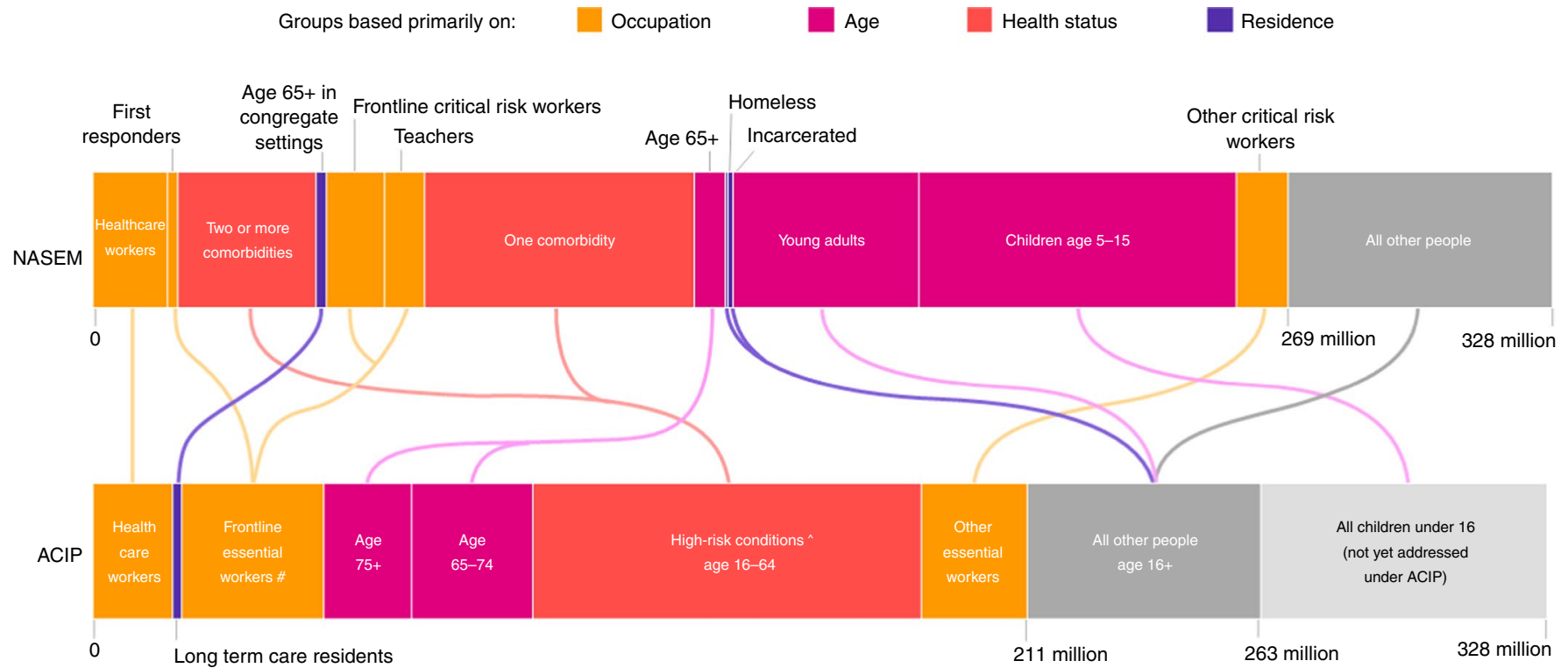

Fig. 1 | Priority groups under NASEM and ACIP frameworks. Group sizes take into account overlaps with preceding groups, so as not to double-count people who fall into more than one priority group. Group sizes vary between NASEM and ACIP because they are defined and constructed differently. NASEM's priority groups cover $82 \%$ of the US population; ACIP's cover $64 \%$, or $80 \%$ of the population over 16 years of age. (for methods, see https://vaccineallocation.ariadnelabs.net/assets/Vaccine_Allocation_Planner_for_COVID19_Methods.pdf; depiction: Ariadne Labs) \#Includes first responders and teachers. Includes pregnancy and smoking (not included in NASEM).

another). Five states refer to more than one index. Among the 20 high-disadvantage jurisdictions (where more than $25 \%$ of the local population are among the nationwide most disadvantaged group; Extended Data Fig. 1), 14 report using an index.

Fourteen jurisdictions (three cities and 11 states) describe zip code-based prioritizations combining the US Post Office geographic structure with different proxies for disadvantage. In several cases, more than one proxy was mentioned. By frequency, we found zip code in combination with COVID-19 incidence $(n=5)$; vaccine uptake rates $(n=4)$, COVID-19-associated mortality $(n=3)$; bespoke algorithms developed by the respective health department $(n=2)$; economic data $(n=2)$; general health data $(n=2)$; hospitalization rates $(n=1)$; and social data $(n=1)$. In five cases, the metric applied to the zip code was unclear. Colorado focuses on census tracts with the highest density of low-income and minority communities (Table 3 and Supplementary Data).

Thirty-six jurisdictions indicate using Tiberius (which, in principle, could enable applying SVI-weights to allocations), including 13 jurisdictions not otherwise signaling any use of a place-based measure.

Among the 34 states and three cities that refer to a disadvantage index, five different purposes can be distinguished. (Some jurisdictions indicate the intention to pursue more than a single goal; see overviews in Table 3 and full data in Supplementary Table 1).

In direct alignment with NASEM's recommendation, 25 total jurisdictions (17 using SVI, seven using zip code and one using census tracts) use language that makes it clear that a place-based measure not only has public health relevance but also can be used for promoting equity. Expressly, they refer to the need to address existing inequities across racial, ethnic and income groups that are associated with poverty, deprivation and differential COVID-19 impact and burden in health and economic terms, that live in underserved areas or that belong to a historically or systematically marginalized population (AR, CA, CHI, CO, CT, DC, Houston, IN, IL, LA, MA, MI, MD, MN, NC, ND, NH, NM, NV, NYC, OH, Philadelphia, TN, $\mathrm{TX}$ and $\mathrm{WI})^{12,30}$. Specific policy responses are found in jurisdictions specifying increased allocations ${ }^{30}$ or larger shares of appointments for people from more disadvantaged areas. Tennessee, the first state to mirror NASEM's approach at the state level, reserves $5 \%$ of its Moderna vaccine allocation for high-SVI areas. New Hampshire adds $10 \%$ of its allocation to communities disproportionately impacted, drawing on SVI and CCVI. Massachusetts allocates 20\% additional vaccines to communities with disproportionate COVID19 burden and high social vulnerability. Connecticut commits to administering at least $25 \%$ of available vaccine supply to high-SVI areas. California reserves $40 \%$ of vaccines for communities in the first quartile of its index and recommends that a similar share be reserved in appointments. North Carolina reserves 30\% of vaccines for purposes including equitable access for racial and ethnic minorities and requests that $40 \%$ of daily vaccinations be reserved and filled with individuals from historically marginalized populations first. Arkansas and Illinois use the CCVI to apply unspecified weights to increase allocations for more disadvantaged communities. Indiana, Michigan, Minnesota, North Dakota, Ohio and Wisconsin indicate the same, using SVI.

Eighteen jurisdictions plan to use an index to identify priority populations (AL, AK, FL, GA, Houston, KS, MD, NY, OR, PA, RI, SC, TX, VA, VT, WA and Philadelphia), which might entail increased vaccine or appointment allocations or earlier placement in the sequence of priority groups or other targeted activities.

Fifteen jurisdictions plan to use an index for promoting access: planning locations of dispensing sites (CT, LA, MI, NC, NH, NJ, Philadelphia and SD) or outreach or communication strategies (AK, AZ, CT, LA, MA, MD, MI, NC, NY, VT, WA and Philadelphia).

Finally, four states states (CA, MI, NC and $\mathrm{OH}$ ) use an index to monitor vaccine receipt. North Carolina set the goal that minority populations should receive vaccines at least proportionate to their population share, and Michigan aims to have no differences across racial, ethnic or SVI groups.

\section{Discussion}

We highlight four aspects regarding our appraisal of the data and their broader implications: (1) variation among jurisdictions in the adoption of disadvantage indices; (2) variations in the types 
Table 2 | Jurisdictions' use of disadvantage indices and zip codes for prioritizing vaccine allocation and use of Tiberius software

\begin{tabular}{|c|c|c|c|c|c|c|c|c|c|c|c|c|}
\hline & \multicolumn{8}{|c|}{ Among all 64 jurisdictions } & \multicolumn{4}{|c|}{$\begin{array}{c}\text { Among the } 56 \text { jurisdictions } \\
\text { with SVI data }\end{array}$} \\
\hline & \multicolumn{2}{|c|}{ Total $^{\mathrm{b}}$} & \multicolumn{2}{|c|}{$\begin{array}{l}50 \text { states } \\
\text { and DC }\end{array}$} & \multicolumn{2}{|c|}{ Cities } & \multicolumn{2}{|c|}{ Territories } & \multicolumn{2}{|c|}{$\begin{array}{l}\text { High } \\
\text { disadvantage } \\
\text { (SVI) }\end{array}$} & \multicolumn{2}{|c|}{$\begin{array}{l}\text { Low } \\
\text { disadvantage } \\
\text { (SVI) }\end{array}$} \\
\hline & $n$ & $\%$ & $n$ & $\%$ & $n$ & $\%$ & $n$ & $\%$ & $n$ & $\%$ & $n$ & $\%$ \\
\hline Total $n$ & 64 & & 51 & & 5 & & 8 & & 20 & & 36 & \\
\hline Using zip code & 14 & $22 \%$ & 11 & $22 \%$ & 3 & $60 \%$ & 0 & $0 \%$ & 9 & $45 \%$ & 5 & $14 \%$ \\
\hline Using Tiberius & 36 & $56 \%$ & 34 & $67 \%$ & 0 & $0 \%$ & 2 & $25 \%$ & 8 & $40 \%$ & 26 & $72 \%$ \\
\hline \multicolumn{13}{|l|}{ Among those using any index: } \\
\hline Total $n$ & 37 & & 34 & & 3 & & 0 & & 14 & & 23 & \\
\hline \multicolumn{13}{|l|}{ Index used for: ${ }^{b}$} \\
\hline More vaccines/appointments & 17 & $46 \%$ & 16 & $47 \%$ & 1 & $33 \%$ & 0 & & 6 & $43 \%$ & 11 & $48 \%$ \\
\hline Defining priority groups, areas \pm prioritize people & 17 & $46 \%$ & 15 & $44 \%$ & 2 & $67 \%$ & 0 & & 7 & $50 \%$ & 10 & $43 \%$ \\
\hline Plan outreach, communication & 12 & $32 \%$ & 11 & $32 \%$ & 1 & $33 \%$ & 0 & & 4 & $29 \%$ & 8 & $35 \%$ \\
\hline Planning dispensing sites & 8 & $22 \%$ & 7 & $21 \%$ & 1 & $33 \%$ & 0 & & 3 & $21 \%$ & 5 & $22 \%$ \\
\hline Monitoring receipt & 4 & $11 \%$ & 4 & $12 \%$ & 0 & $0 \%$ & 0 & & 2 & $14 \%$ & 2 & $9 \%$ \\
\hline \multicolumn{13}{|l|}{ Among those using any zip code: } \\
\hline Total $n$ & 14 & & 11 & & 3 & & 0 & & 9 & & 5 & \\
\hline \multicolumn{13}{|l|}{ Zip code used for:b } \\
\hline More vaccines/appointments & 7 & $50 \%$ & 4 & $36 \%$ & 3 & $100 \%$ & 0 & & 6 & $67 \%$ & 1 & $20 \%$ \\
\hline
\end{tabular}

${ }^{2}$ Excludes the eight territories owing to lack of SVI data. For construction of high- and low-disadvantage jurisdictions, see Supplementary Table 2. ${ }^{\text {b }}$ Column can sum to over $100 \%$ because jurisdictions could be placed in more than one category. For details on which jurisdiction makes use of what index, see Supplementary Table 1.

of uses to which such measures are put; (3) plans for the uptake of the Tiberius software; and (4) the importance of monitoring. Additionally, we note implications for adoption beyond the United States.

By March 2021, 6 months after first being asked to publish their formal allocation frameworks, 37 jurisdictions and most states $(n=34)$ had adopted a disadvantage index. Including zip code-based measures, roughly two-thirds of jurisdictions $(n=43)$ were using a place-based measure of disadvantage. The dominance of disadvantage indices over zip code approaches is likely explained by NASEM's recommendation to use the SVI or a similar index and by the fact that combining zip codes with proxy measures for disadvantage incurs a justificatory burden for the metric chosen. Although the rapid pace of adoption is remarkable, it is not universal across jurisdictions. Place-based disadvantage measures are not the only way equity could be addressed, and jurisdictions might have alternative strategies. But scrutiny of efforts to implement ways of allocating vaccines in ways that reduce inequities will likely increase. For example, even if all states were to set aside a $10 \%$ reserve of their allotted vaccines as additional amounts for the most disadvantaged quartile, under the NASEM framework populations of color would be offered vaccines below their population share until the beginning of phase 3, except for the very first phase (see Extended Data Fig. 3, simulation for ACIP framework ongoing) ${ }^{3}$.

An important use of place-based disadvantage measures relates to the expression among vaccine workers that 'Vaccines don't save lives. Vaccinations save lives. ${ }^{31}$ Setting aside larger shares of vaccines alone can be meaningless for reducing inequity if these steps are not matched with genuine and proactive efforts to make vaccines available in conveniently located and trusted settings. However, currently, only 18 jurisdictions indicate using a disadvantage index for planning the location of vaccination sites or communication and outreach efforts.

Using a rigorous measure of disadvantage for promoting receipt is of great importance in view of the overall policy that jurisdictions receive only new vaccine allocations once already received batches have been distributed ${ }^{32}$. Although entirely reasonable in its motivation to increase population protection, an unintended consequence of this policy can be that jurisdictions might prioritize regions where uptake is swift and virtually guaranteed ${ }^{33}$ and, conversely, might deprioritize locations with real or anticipated lower receipt. But interpreting low vaccine receipt in, for example, communities with predominantly Black, Hispanic or Indigenous populations as expressing that these groups might simply not be interested in 
Table 3 | Central verbatim sections illuminating jurisdictions' uses of disadvantage indices and zip codes in combination with proxies for disadvantage, to promote equitable vaccine allocation

Prioritize disadvantaged groups though larger allocations, share of vaccination appointments $(n=13 / 24)$

CA The state is using the Healthy Places Index (HPI), which reflects 25 community characteristics using data related to the economy, education, healthcare access, housing, neighborhoods, clean environment, transportation and social environment. California's 1,650+ ZIP codes have been divided into four quarters based on the HPI Index. [...]

Allocation Formula... first doses will be allocated based on two steps:

1. Eligible Population: $70: 30$ based on the geographic distribution of the population eligible for the vaccine, with 70 percent based on age eligibility and 30 percent based on sector eligibility.

2. Double Allotment for Lowest HPI Quartile: Next based on the 70:30 split, zip codes in the first HPI quartile (that is, those with the lowest 25 percent HPI scores) will be allocated 40 percent of the state's available vaccine doses. Since approximately 40 percent of COVID cases and deaths are in the first HPI quartile, a similar percentage of doses should be administered in these communities.

CT Efforts to increase equity in vaccine distribution to residents of underserved communities that have been hardest hit by COVID-19, the Connecticut Department of Public Health has identified the individual zip codes across the state that are in the top 25 percent of the CDC's social vulnerability index (SVI). The department is working with vaccine providers to commit to administering at least 25 percent of available vaccine supply to the residents living in these priority zip codes and to collecting, tracking and reporting data regarding race and ethnicity for vaccines administered.

DC Beginning next week, the District will switch over to a pre-registration system for making vaccination appointments. Under the new system, individuals will be able to provide their information to DC Health through a pre-registration website or by calling the District's call center. As appointments are made available, individuals who have pre-registered will receive an email, phone call and/or text message alerting them that they have an opportunity to make a vaccination appointment. Equity will continue to be a top priority, and DC Health will continue to set aside appointments for residents living in priority zip codes. [...] Priority zip codes include areas of the city where residents are disproportionately affected by COVID-19, including number of cases and mortality rate.

Hou SPL2: The Houston Health Department's strategy for the NRG Park site will utilize its Area Agency Aging waitlist with the following prioritization criteria:

Priority 1 - People age 65 and older who live in a high-risk zip code,

Priority 2 - People age 65 years and older,

Priority 3 - People age 60-64 with underlying medical conditions who live in high-risk zip codes, and

Priority 4 - People age 60-64 with underlying medical conditions.

Houston's high-risk zip codes are geographic areas with people more vulnerable to severe COVID-19 illness as identified by positivity rate, underlying health condition and economic and social data.

IL IDPH will estimate overall allocations of COVID-19 vaccine based on the size of critical population groups within each local public health department's jurisdiction and weighted using the COVID-19 Community Vulnerability Index (CCVI).

In advance of the full activation of the Federal Retail Pharmacy Program, Illinois will transfer a portion of the state's allocation to partnering pharmacies and direct allocations to areas identified as more vulnerable through the COVID-19 Community Vulnerability Index (CCVI).

Appointments will also be set aside for Chicago residents who live in high CCVI (COVID Community Vulnerability Index) ZIP codes and/or ZIP codes with low vaccination rates, allowing anyone from these ZIP codes to register. More information will be provided on eligible ZIP codes and the subsequent registration process later this week.

MD As the first mass vaccination center in Baltimore, BCCFH has been focused on developing proactive and adaptable approaches to expanding access to COVID-19 vaccines among communities in Baltimore where vaccine uptake is lagging. BCCFH leadership has worked closely with hospital-based community health teams to actively encourage eligible individuals who live in high-vulnerability ZIP codes to register for vaccination. Using a custom algorithm, the BCCFH has worked to ensure that over $40 \%$ of recipients eligible for vaccination at $\mathrm{BCCFH}$ were Baltimore City residents, prioritizing individuals in vulnerable communities first.

MN For Phase 1a, vaccine doses will be allocated to every region in Minnesota based on two factors:

1. Population of health care personnel and long-term care facility residents within each region.

2. Population residing in vulnerable census tracts identified by the Social Vulnerability Index (SVI)

Phase 1a Allocation: $15 \%$ of doses allocated based on SVI. $85 \%$ pro rata allocated based on priority population.

NC Holding slots for HMPs • Out of 100 daily slots, reserve 40 to ensure they are filled with individuals from HMP first. [...] After reserving the 89,550 doses for enrolled providers, that leaves an estimated 37,575 doses (based on continuing to receive 127,125 doses per week) to prioritize for several efforts [including] increased vaccine for counties to account for larger historically marginalized populations and larger populations over 65 [and providing vaccines for] projects and events that promote increased access and partnerships in the community, with particular focus on achieving racial and ethnic equitable access to vaccine. The percentage of vaccine administered to historically marginalized and minority populations should meet or exceed the population estimates of these communities in their county and region.

ND The ND Advisory Committee on COVID-19 Vaccine Ethics may choose to utilize CDC's vulnerability index when allocating vaccine, which may ensure equity in the number of doses Tribal healthcare providers receive. 
Table 3 | Central verbatim sections illuminating jurisdictions' uses of disadvantage indices and zip codes in combination with proxies for disadvantage, to promote equitable vaccine allocation (Continued)

Prioritize disadvantaged groups though larger allocations, share of vaccination appointments $(n=13 / 24)$

$\mathrm{NH} \quad \mathrm{NH}$ will allocate $10 \%$ of the available state vaccine supply to geographic areas that are highly vulnerable to COVID-19. DPHS will:

- Identify communities through the COVID-19 Community Vulnerability Index (CCVI) and US Census data

- Distribute vaccines predominately through mobile vaccination clinic sites

- Coordinate this distribution through the NH Regional Public Health Networks at sites that are (1) familiar and accessible to the target population and (2) geographically positioned for easy access on foot or through public transportation when available.

- Initially provide vaccine to NH's racial and/or ethnic minority community then include other vulnerable populations, such as those that are geographically isolated or those living in economic hardship.

- Reserve vaccine for use in targeted response in these identified census tract areas if needed.

The CCVI identifies communities within the context of the COVID-19 pandemic that may be more vulnerable than others due to limited ability to mitigate, treat, and delay transmission of a pandemic disease, and to reduce its economic and social impacts.

NM To ensure allocation equity, we have:

- Added an equity-based component to our allocation system based on the CDC's Social Vulnerability Index (SVI) and COVID-19+ rates, reallocating up to $25 \%$ of vaccine supply each week to populations with a high SVI and/or >10,000 COVID-19 cases per 100,000 population

NYC To deepen this work, City will now prioritize appointments for residents at vaccine sites in the Taskforce neighborhoods, setting aside specific hours and slots. [...] Building on the initial list of 27 neighborhoods, the Taskforce has broadened its criteria and expanded to a total of 33 neighborhoods, ... based on a range of factors including high COVID-19 mortality and case rates, high prevalence of chronic illness, presence of overcrowded housing, the number of individuals experiencing poverty, and other preexisting health disparities.

WI [I]t is appropriate to allocate resources to areas or regions disproportionately burdened by COVID-19 and other health inequities with the following goals:

1. Reduce the higher rates of severe COVID-19 illness and mortality being experienced by systematically disadvantaged social groups and marginalized populations.

2. Address disproportionate economic and social impacts on some population groups, especially those that are marginalized or systematically disadvantaged.

Strategies and tools to account for disadvantages that increase risk for COVID-19 should be considered. Examples include the Centers for Disease Control and Prevention's (CDC's) Social Vulnerability Index and the Area Deprivation Index.

Define priority groups, areas, possibly also prioritize $(n=7 / 17)$

AK The State of Alaska Covid-19 Vaccine Task Force implementation teams use tools such as the area deprivation index (ADI) to identify specific geographic areas within communities that experience higher levels of deprivation compared to surrounding areas.

Hou In addition to the age cohorts, HCPH will be prioritizing areas where there have been COVID health disparities (using the CDC's social vulnerability index and where data shows there are [...] increased COVID cases or deaths and/or COVID undertesting).

MD The VETF is guided by the careful use of recognized socioeconomic variables to identify communities and individuals who are vulnerable, under-served, hesitant, or difficult-to-reach. Key variables include: - Population over 65 years old - Population with an annual income below $\$ 49,000$ - Unemployment rate - Population older than 25 years old without a high school diploma -Minority composition of the community -Single parent households -Housing with more than one person per room -Households without access to a vehicle -Total COVID cases Amount of population receiving at least the first dose ... and the Center for Disease Control and Prevention's Social Vulnerability Index.

NY Once the vaccine is first approved for use, New York State will use up-to-date data to determine which geographic areas of the state may derive a greater public health benefit to receiving early vaccine. This may include areas with higher historical burden of disease or areas that have the highest prevalence of COVID-19. [...] New York will also consider whether the vaccine can be used effectively as a potential outbreak interruption strategy and if so, what the criteria will be.

OR Options for mapping population data (including Tiberius, Tableau and ArcGIS) are actively being explored in conjunction with mapping of CDC's Social Vulnerability Index (SVI) to identify overlap and potential areas of greatest need.

VA We are also identifying zip codes with the greatest number of COVID-19 cases and deaths and prioritizing neighborhoods with higher Social Vulnerability Index scores.

VT The Immunization Program will work closely with all COVID-19 vaccination providers and target settings to ensure equitable access to the COVID-19 vaccine. Vaccine allocation will be based on population data, with attention to critical populations. Vaccine administration data from the Immunization Registry will be monitored and reviewed by geographic location. Vaccine doses administered by enrolled sites will also be monitored and redistribution will be required. The Immunization Program is collaborating with the Health Operations Center's Health Equity and Community Engagement Team to ensure access for people who are disproportionately affected by COVID-19, including Black, Indigenous and people of color. GIS mapping and Social Vulnerability Indices will be employed to identify areas with limited access and direct distribution efforts.

Plan outreach/communication $(n=7 / 15)$

AK The State of Alaska Covid-19 Vaccine Task Force implementation teams use tools such as the area deprivation index (ADI) to identify specific geographic areas within communities that experience higher levels of deprivation compared to surrounding areas. DHSS implementation teams are working with a range of stakeholders to focus outreach efforts to ensure equitable access to vaccines for people in each phase. 
Table 3 | Central verbatim sections illuminating jurisdictions' uses of disadvantage indices and zip codes in combination with proxies for disadvantage, to promote equitable vaccine allocation (Continued)

Prioritize disadvantaged groups though larger allocations, share of vaccination appointments $(n=13 / 24)$

CT [To] efforts to increase equity in vaccine distribution to residents of underserved communities that have been hardest hit by COVID-19, the Connecticut Department of Public Health has identified the individual zip codes across the state that are in the top 25 percent of the CDC's social vulnerability index (SVI). [The] equity pledge calls for providers to make investments in outreach and access efforts to reach vulnerable communities, particularly communities of color, including dedicated call-in lines, reserved appointment slots for priority zip codes, community health worker models, and mobile clinics in priority neighborhoods.

MA This list of 20 is a subset of the cities and towns that met the Massachusetts COVID Advisory Group recommendation to prioritize communities using the CDC's Social Vulnerability Index (SVI) and COVID-19 case rates since the start of the pandemic. Those communities were then ranked by average daily COVID-19 case rates in each city and town (excluding cases in long-term care and correctional facilities and communities with under 30,000 residents). From this ranked list by case rate, the top 17 cities and towns with the highest percentage of people of color were identified. The list of 20 cities and towns includes three additional communities to capture the top 15 communities with the highest daily COVID case rates.

MI The SVI indicators help to prioritize distribution of scarce resources such as COVID-19 testing sites and vaccinations to ensure effectiveness and equity for all Michiganders. Indicators like poverty and transportation can highlight places where people may have difficulty accessing COVID19 testing, treatment and vaccination. While supplies are low, outreach efforts must be concentrated in areas with higher social vulnerabilities, where people may face more difficulty accessing vaccine. Vaccine administrators should use the SVI to develop targeted outreach strategies while planning vaccine clinics to ensure protection of people who are the most vulnerable.

NC The percentage of vaccine administered to historically marginalized and minority populations should meet or exceed the population estimates of these communities in their county and region. Please see the specific operational considerations below to ensure you are deploying best practices to meet this expectation. Providers should engage in partnerships, targeted outreach and vaccine events to vaccinate historically marginalized populations and meet this goal.

NY Persons in areas that have a high social vulnerability index are particularly vulnerable to COVID-19 and should be notified about how they can receive vaccine.

RI Rhode Islanders of color and those living in specific geographies have had higher rates of COVID-19 infections, hospitalizations, and deaths throughout the pandemic. For example, in the seven hardest-hit ZIP codes, people as young as 25 are experiencing higher hospitalization rates than people over 60 years old in the rest of the state. These differences are rooted in systemic racism, which has caused obstacles to health in the form of insufficient access to things like quality education and housing, good jobs with fair pay, safe environments and access to healthcare. [Focused] efforts to expand access to and uptake of vaccine among communities of color at highest risk of hospitalization and death [are therefore needed] to ensure vaccine access for the most vulnerable members of the hardest-hit areas.

Plan dispensing sites $(n=5 / 11)$

$\mathrm{CT} \quad$ Points of distribution are being mapped and planned against CDC's social vulnerability index

LA Through geospatial mapping to the zip code level, the state will continue to ensure the sites providing vaccine are appropriately located geographically and meeting the needs of the communities in which they are located. If there are areas identified as needing additional vaccine availability, OPH will further recruit and enroll sites as needed. Additionally, the state will have the capacity to deploy Mobile Vaccination Teams to provide vaccination services in areas where any gaps in access are identified. Targeted messaging may also be directed to areas with low vaccine update..

$\mathrm{NH} \quad$ DPHS will:

- Identify communities through the COVID-19 Community Vulnerability Index (CCVI) and US Census data

- Distribute vaccines predominately through mobile vaccination clinic sites

- Coordinate this distribution through the NH Regional Public Health Networks at sites that are (1) familiar and accessible to the target population and (2) geographically positioned for easy access on foot or through public transportation when available.

- Initially provide vaccine to NH's racial and/or ethnic minority community then include other vulnerable populations, such as those that are geographically isolated or those living in economic hardship.

- Reserve vaccine for use in targeted response in these identified census tract areas if needed.

NJ Social Vulnerability Index (SVI) to determine location of PODS [points of dispensing]

OK Efforts are being made to identify and enroll pandemic providers in counties and zip codes with socially vulnerable populations. Federally qualified health centers are an important partner to administer COVID 19 vaccine to minority population. If the tribes decide to receive state allocation, tribal providers and other providers serving tribes will be enrolled as pandemic providers.

Monitor receipt $(n=4 / 9)$

CA (Vaccine Equity Metric) This graph compares COVID-19 vaccinations among four different levels of community health. It uses the Public Health Alliance of Southern California's Healthy Places Index (HPI) measures in a zip code area that can impact health, like income, education, and access to health care. Areas are then given a score, ranging from least healthy community conditions (Quartile 1) to most healthy community conditions (Quartile 4).... Data below show the progress of vaccination in each of the four quartiles.

MI [Under goals] Zero Disparity. There is no disparity in vaccination rates across racial and ethnic groups or by social vulnerability index. [Get more people vaccinated]: Utilize Social Vulnerability Index in vaccination process.

$\mathrm{OH} \quad$ In addition, vaccine administration will be assessed using the CDC's Social Vulnerability Index both a priori when deciding geographic distribution of vaccines and post-hoc to ensure that state's goals to protect the most-at-risk and vulnerable Ohioans are upheld.

SC SIMON contains vaccine coverage report capabilities and can stratify reporting variables by geographic region down to the zip code level. Vaccine coverage reports will be automatically generated weekly and disseminated to the Immunization Branch director and Regional Clinic Coordinators to review to determine gaps in vaccination coverage and uptake.

Selection: For a full summary of all types of use, and all extracted data, see Supplementary Data. $n$ refers to use for both disadvantage index and zip codes. 


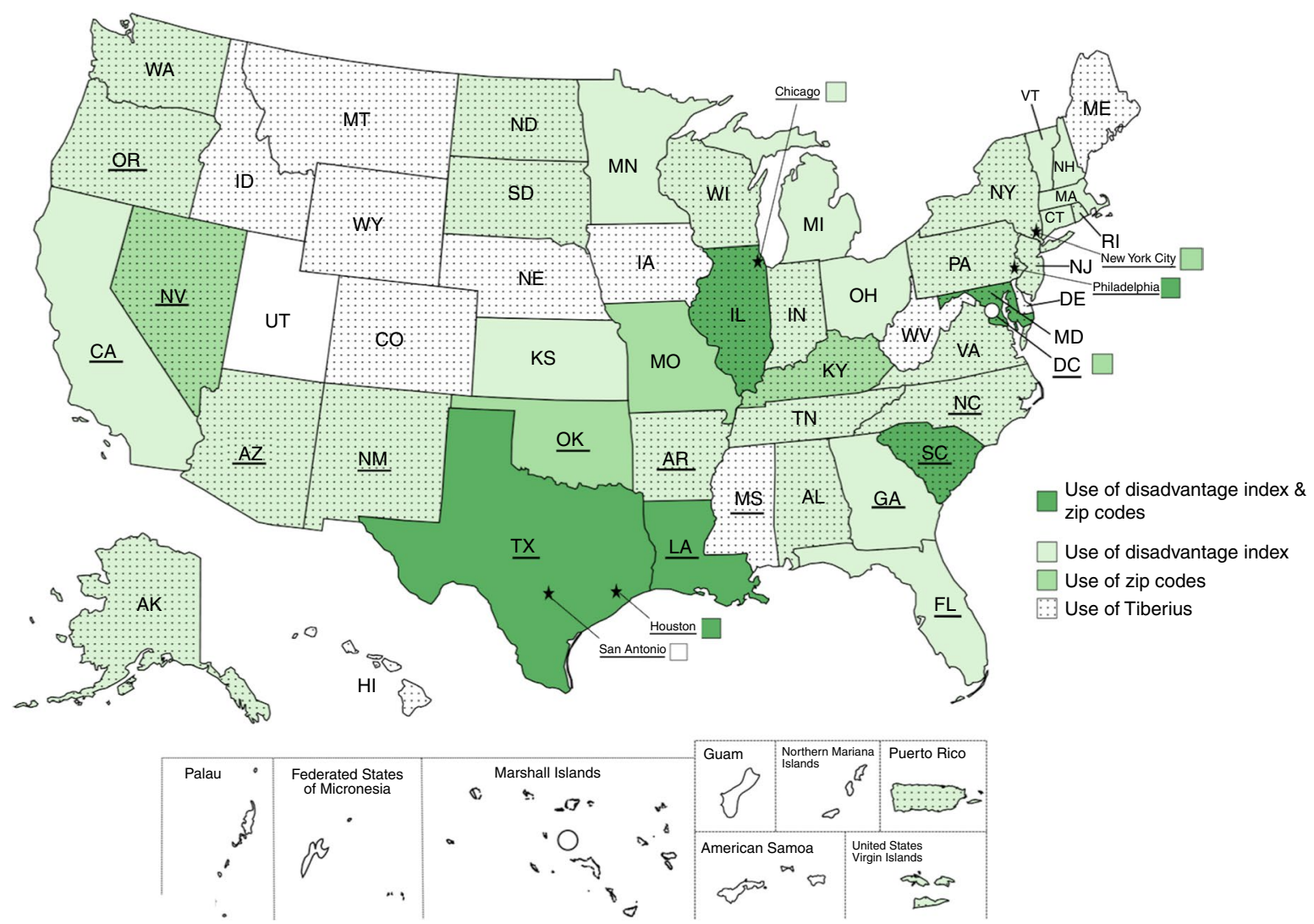

Fig. 2 | Jurisdictions' use of disadvantage indices and zip codes for prioritizing vaccine allocation and use of Tiberius software-a geographical depiction. Underline indicates that the state (including DC) is among the 20 with the largest share of disadvantaged communities. For construction of high- and low-vulnerability jurisdictions, see Supplementary Table 2.

vaccines due to personal reasons is shortsighted. Lacking trust in the healthcare system or government, and ongoing experiences of structural racism in healthcare and beyond, can be powerful barriers to vaccine uptake $e^{24,26,27,34}$. Likewise, given that the intention to be vaccinated among Black Americans has approximated that of white and Hispanic Americans (61\% versus 69\% versus $70 \%)^{35}$, lower rates of receipt by Black Americans could plausibly reflect insufficient opportunities to receive vaccines in a trusted setting ${ }^{36}$. Given that current incentive structures implicitly favor prioritizing allocations to geographic areas with the swiftest uptake, using place-based measures for targeted outreach, communication, appointment sign-up assistance and dispensing site planning are, therefore, critical, especially in jurisdictions with larger proportions of disadvantaged communities of color and others disengaged from healthcare systems. Such measures can also help mitigate the fact that populations who are more 'internet savvy' gain advantages in allocation systems that often rely on online reservations for vaccines ${ }^{37}$.

On a practical note (that can have normative implications), use of the Tiberius software is not universal among the jurisdictions. In principle, uniform adoption of a centralized platform to inform state plans could be helpful. Two main uses of Tiberius are consistent implementation of index-based prioritization by disadvantage and near-real-time transparency around vaccine allocation and receipt. However, Tiberius seems mainly to represent an opportunity missed, as it appears that policymakers engaging with the software had major concerns, including around the opacity of data integration and alignment of data representation with state-level datasets $^{38}$.

As jurisdiction-level planners distribute vaccines in weekly batches, each delivery offers an opportunity to monitor the status of vaccine receipt by vulnerable populations and course correct when and where needed. Targets such as Michigan's Zero Disparity goal (achieving no disparity in vaccination rates across racial and ethnic groups or by social vulnerability index) provide critical orientation and succinctly articulate a central notion of health equity that aims to allocate resources not per capita alone but also in ways that avoidable unfair differences in health outcomes are genuinely addressed. Monitoring matters throughout all allocation phases. It also gains in importance at the point where rationing seemingly ends (once vaccines are offered to the entire population). Making all residents eligible is not the same as getting everyone vaccinated. Scarcity of availabile vaccines decreases gradually, and the switch to the final phase (universal eligibility) marks a step change. Equity issues, therefore, continue to persist, as the general public, just as in all previous phases, is not a homogenous group and differs in their risks of getting and spreading the virus. Monitoring receipt (and intensifying, as appropriate, outreach, communication or dispensing site efforts) is especially important for jurisdictions that open eligibility to the general population but have below-average vaccination rates and above-average proportions of disadvantaged communities ${ }^{39}$.

Finally, although the United States stands out globally in the magnitude of disparities across racial, ethnic and income groups relative to the country's wealth, it is certainly not the only country that struggles with inequities. As noted, WHO guidance expressly urges planners around the world to consider ways of allocating vaccines equitably within nations ${ }^{13}$. Our review was restricted to the United States but can raise the question of how feasible the adoption of disadvantage indices or other place-based measures would be in other countries. A particularly striking example is the United Kingdom (UK), which was one of the first countries to establish and implement such measures in health policy more broadly ${ }^{40}$. The UK's Indices of Multiple Deprivation have been used for directly related 


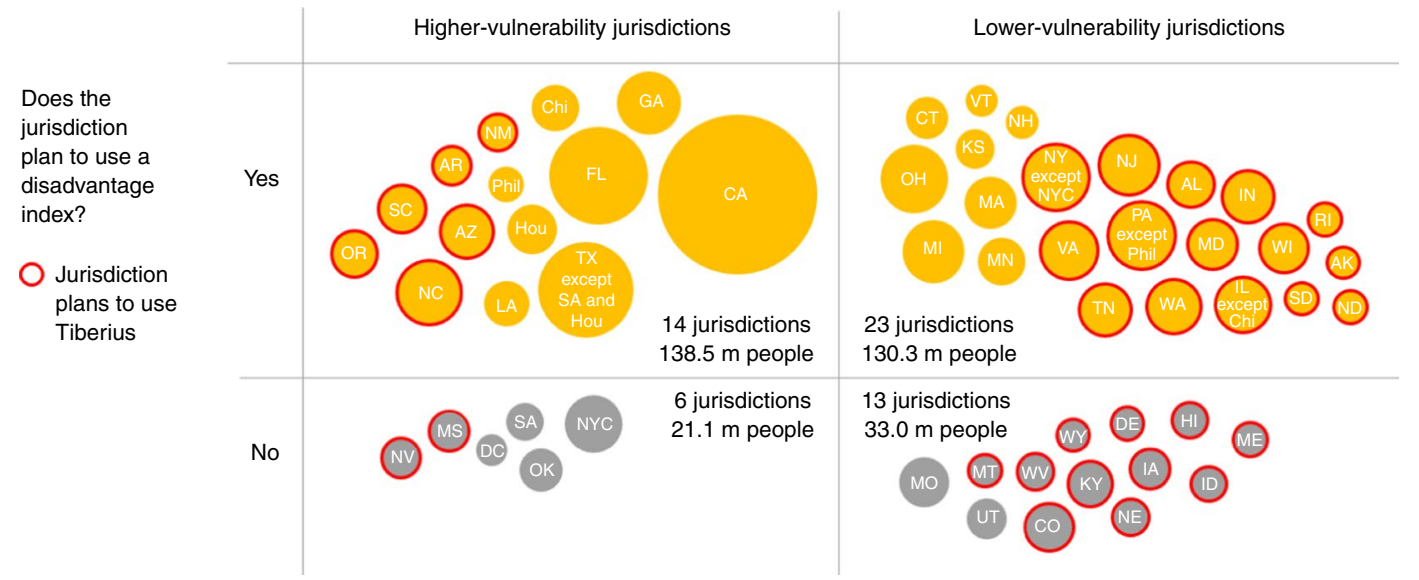

Fig. 3 | 56 Jurisdictions' use of disadvantage indices, by share of population in vulnerable areas, population size and use of Tiberius software. Circle size is proportional to jurisdiction population. All population data includes people under 16 years of age (not currently incorporated in ACIP's framework). Higher vulnerability $=25 \%$ or more of the jurisdiction's population lives in census tracts with SVI scores in the most disadvantaged quartile (Extended Data Fig. 1). Phil, Philadelphia PA; SA, San Antonio TX; Chi, Chicago IL; Hou, Houston TX; NYC, New York City NY. Excludes the eight territories owing to lack of SVI data. (Depiction: Ariadne Labs). m, milllion.

budgetary resource allocations, and evaluations demonstrated their effectiveness at reducing disparities ${ }^{40}$. However, the UK's allocation framework makes no use of it, despite an express acknowledgment of clear evidence demonstrating marked disparate impact in incidence and mortality across racial, ethnic and income groups ${ }^{41}$. At the same time, we note that a new vulnerability index specifically for guiding COVID-19-related allocation has been developed for India ${ }^{42}$. The different ways in which US planners are using place-based disadvantage measures could, therefore, be helpful for vaccination planning outside of the United States, particularly in countries with similarly pronounced patterns of disparities in health, wealth and COVID-19 impact.

Our study had some limitations. Jurisdictions were asked to publish initial allocation plans under a constrained schedule with only 30 days between the official request and the deadline. Although summary allocation plans were eventually available for all jurisdictions, full allocation plans were publicly available for $81 \%$ of jurisdictions as of 31 October 2020. After vaccine rollouts started, some jurisdictions stopped updating or publishing their original documents, and not every intended use of place-based measures might have been captured. At the same time, our analysis includes all states and demonstrates that an increasing number of jurisdictions have adopted NASEM's recommendation to use a disadvantage index alongside a primarily risk-based framework with sequential subpopulations, despite disadvantage indices going unmentioned by ACIP. The data presented here cannot settle exhaustively the extent to which jurisdictions use indices primarily to reduce inequity (that is, in full alignment with NASEM's social justice rationale) or for less normative reasons, which might be grounded more in epidemiology. Still, our analysis provides a historical benchmark in that at least 22 states, DC and three cities expressly note social justice considerations in explaining their use of an index (Table 2 and Supplementary Data), and, hence, indicate that reducing inequity through the use of place-based disadvantage measures is perceived to be both pressing and feasible relative to other important priorities.

The United States continues to face an unprecedented public health, logistical and social justice challenge in allocating vaccines $^{43,44}$. In a major shift in designing rationing frameworks, most US states have recognized the need to promote equity within allocation phases through the use of place-based disadvantage indices and related measures. Although ongoing impatience with delays in receiving vaccines is understandable, within each priority group some communities remain more able to protect themselves from COVID-19 than others. Jurisdictions should explore, to the fullest extent, the potential of using disadvantage indices alongside other options to allocate vaccines equitably, not just within each of the priority populations but also now that vaccines are offered to the general population ${ }^{4,12}$

Although the tasks at hand are urgent and dynamic, there is still time for jurisdiction planners to play a direct role in changing the course of a troubling historical trajectory of inequity. Allocation frameworks that increase the chances of more disadvantaged communities-and particularly those of color-to be offered a vaccine can help to reduce inequity and promote public health simultaneously $y^{6,7}$ and can be one way of mitigating the consequences of past, and, in many ways, still ongoing ${ }^{9,26-28,34,36}$, wrongs.

\section{Online content}

Any methods, additional references, Nature Research reporting summaries, source data, extended data, supplementary information, acknowledgements, peer review information; details of author contributions and competing interests; and statements of data and code availability are available at https://doi.org/10.1038/ s41591-021-01379-6.

Received: 12 March 2021; Accepted: 29 April 2021;

Published online: 18 May 2021

\section{References}

1. Centers for Disease Control and Prevention (CDC). COVID Data Tracker. https://covid.cdc.gov/covid-data-tracker/\#datatracker-home. (accessed 19 April 2021).

2. Centers for Disease Control and Prevention (CDC). COVID-19 Vaccination Program Operational Guidance. https://www.cdc.gov/vaccines/covid-19/ covid19-vaccination-guidance.html (accessed 8 November 2020).

3. Schmidt, H. et al. Rationing safe and effective COVID-19 vaccines: allocating to states proportionate to population may undermine commitments to mitigating health disparities. Preprint at https://ssrn.com/abstract=3729069 (2020).

4. National Academies of Sciences, Engineering and Medicine. A Framework for Equitable Allocation of Vaccine for the Novel Coronavirus. https://www. nationalacademies.org/our-work/a-framework-for-equitable-allocationof-vaccine-for-the-novel-coronavirus (National Academies of Sciences, Engineering and Medicine, 2020).

5. Berwick, D. M. The moral determinants of health. J. Am. Med. Assoc. 324, 225-226 (2020).

6. Dasgupta, $\mathrm{S}$. et al. Association between social vulnerability and a county's risk for becoming a COVID-19 hotspot-United States, June 1-July 25, 2020. MMWR Morb. Mortal. Wkly Rep. 69, 1535-1541 (2020). 
7. Karmakar, M., Lantz, P. M. \& Tipirneni, R. Association of social and demographic factors with COVID-19 incidence and death rates in the US JAMA Netw. Open. 4, e2036462 (2021).

8. Khazanchi, R., Evans, C. T. \& Marcelin, J. R. Racism, not race, drives inequity across the COVID-19 continuum. JAMA Netw. Open. 3, e2019933 (2020)

9. Yearby, R. \& Mohapatra, S. Law, structural racism, and the COVID-19 pandemic. J. Law Biosci. 7, lsaa036 (2020).

10. Nancy McClung, M. C. et al. The Advisory Committee on Immunization Practices' ethical principles for allocating initial supplies of COVID-19 vaccine-United States, 2020. MMWR Morb. Mortal. Wkly Rep. 69, 1782-1786 (2020).

11. Bell, B. P., Romero, J. R. \& Lee, G. M. Scientific and ethical principles underlying recommendations from the Advisory Committee on Immunization Practices for COVID-19 vaccination implementation. J. Am. Med. Assoc. 324, 2025-2026 (2020).

12. Schmidt, $H$. Vaccine rationing and the urgency of social justice in the Covid-19 response. Hastings Cent. Rep. 50, 46-49 (2020).

13. World Health Organization. WHO SAGE values framework for the allocation and prioritization of COVID-19 vaccination, 14 September 2020. https://apps. who.int/iris/handle/10665/334299 (World Health Organization, 2020).

14. Culyer, A. J. Need-the idea won't do, but we still need it. Soc. Sci. Med. 40, 727-730 (1995).

15. Braveman, P. \& Gruskin, S. Defining equity in health. J. Epidemiol. Community Health 57, 254-258 (2003).

16. Forde, A. T. et al. Discrimination and hypertension risk among African Americans in the Jackson Heart Study. Hypertension 76, 715-723 (2020).

17. Dwyer-Lindgren, L. et al. Inequalities in life expectancy among US counties, 1980 to 2014: temporal trends and key drivers. JAMA Intern. Med. 177, 1003-1011 (2017).

18. Chetty, R., Hendren, N., Jones, M. R. \& Porter, S. R. Race and economic opportunity in the United States: an intergenerational perspective. Q. J. Econ. 135, 711-783 (2019)

19. O’Brien, R., Neman, T., Seltzer, N., Evans, L. \& Venkataramani, A. Structural racism, economic opportunity and racial health disparities: evidence from U.S. counties. SSM Popul. Health 11, 100564 (2020).

20. Adair-Rohani, H. et al. Limited electricity access in health facilities of sub-Saharan Africa: a systematic review of data on electricity access, sources, and reliability. Glob. Health Sci. Pract. 1, 249-261 (2013).

21. Schmidt, H., Pathak, P., Sönmez, T. \& Ünver, M. U. Covid-19: how to prioritize worse-off populations in allocating safe and effective vaccines. BMJ 371, m3795 (2020).

22. Schmidt, H., Gostin, L. O. \& Williams, M. A. Is it lawful and ethical to prioritize racial minorities for COVID-19 vaccines? J. Am. Med. Assoc. 324, 2023-2024 (2020)

23. Schmidt, H. et al. What prioritizing worse-off minority groups for COVID-19 vaccines means quantitatively: practical, legal and ethical implications. Preprint at https://seii.mit.edu/working-paper/what-prioritizing-worse-offminority-groups-for-covid-19-vaccines-means-quantitatively-practical legal-and-ethical-implications/ (2020).

24. Painter, E. M. et al. Demographic characteristics of persons vaccinated during the first month of the COVID-19 vaccination program-United States, December 14, 2020-January 14, 2021. MMWR Morb. Mortal. Wkly Rep. 70, 174-177 (2021).

25. National Strategy for the COVID-19 Response and Pandemic Preparedness. https://www.nhpco.org/wp-content/uploads/White_House_National_Strategy_ for the_COVID-19_Response.pdf (2021).

26. Kakani, P., Chandra, A., Mullainathan, S. \& Obermeyer, Z. Allocation of COVID-19 relief funding to disproportionately black counties. JAMA 324, 1000-1003 (2020).

27. Navathe, A. \& Schmidt, H. Why a hospital might shun a black patient: the way medical professionals are paid keeps structural racism alive. It's unethical and it must change. New York Times https://www.nytimes.com/2020/10/06/ opinion/medical-racism-payment-models.html (6 October 2020).
28. Foster, S., Cannon, Y. \& Bloche, G. Health justice is racial justice: a legal action agenda for health disparities. Health Affairs Blog https://scholarship. law.georgetown.edu/cgi/viewcontent.cgi? article=3317\&context=facpub (2020).

29. Schmidt, H. et al. Equitable allocation of COVID-19 vaccines: an analysis of the initial allocation plans of CDC's jurisdictions with implications for disparate impact monitoring. https://scholarship.law.georgetown.edu/ facpub/2333/ (2020).

30. Pathak, P. A., Sönmez, T., Ünver, M. U. \& Yenmez, M. B. Fair allocation of vaccines, ventilators and antiviral treatments: leaving no ethical value behind in health care rationing. Preprint at https://arxiv.org/abs/2008.00374 (2021).

31. Orenstein, W. Vaccines don't save lives. Vaccinations save lives. Hum. Vaccin Immunother. 15, 2786-2789 (2019).

32. Centers for Disease Control and Prevention (CDC). COVID-19 Vaccination Program Interim Operational Guidance Jurisdiction Operations, Version 2, 29 October 2020. https://www.cdc.gov/vaccines/imz-managers/downloads/ COVID-19-Vaccination-Program-Interim_Playbook.pdf (2020).

33. Buttenheim, A. SARS-CoV-2 vaccine acceptance: we may need to choose our battles. Ann. Intern. Med. https://doi.org/10.7326/M20-6206 (2020).

34. St. Fleur, N. Two Black university leaders urged their campuses to join a Covid-19 vaccine trial. The backlash was swift. STAT https://www.statnews. com/2020/10/12/two-black-university-leaders-urged-their-campuses-tojoin-a-covid-19-vaccine-trial-the-backlash-was-swift/ (October 12, 2020).

35. Johnson, C. \& Funk, C. Black Americans stand out for their concern about COVID-19; 61\% say they plan to get vaccinated or already have. Pew Research Center. https://www.pewresearch.org/fact-tank/2021/03/09/ black-americans-stand-out-for-their-concern-about-covid-1961-say-they-plan-to-get-vaccinated-or-already-have/ (9 March 2021).

36. Ndugga, N. et al. Latest data on COVID-19 vaccinations race/ethnicity. Kaiser Family Foundation. https://www.kff.org/coronavirus-covid-19/issue-brief/ latest-data-on-covid-19-vaccinations-race-ethnicity/ (21 April 2021).

37. Ndugga, N., Artiga, S. \& Pham, O. How are states addressing racial equity in COVID-19 vaccine efforts? Kaiser Family Foundation. https://www.kff.org/ racial-equity-and-health-policy/issue-brief/how-are-states-addressing-racialequity-in-covid-19-vaccine-efforts/ (10 March 2021).

38. Bajak, A. \& Heath, D. A national system to prioritize COVID-19 vaccines has largely failed as states rely on their own systems. USA Today https://www. usatoday.com/story/news/investigations/2021/03/03/covid-19-vaccine-dist ribution-system-fails-live-up-promise/6878303002/ (3 March 2021)

39. Groppe, M. No 'consistent rationale': why vaccinations are open to all in some states and not others. https://money.yahoo.com/ no-consistent-rationale-why-vaccinations-080017243.html (29 March 2021).

40. Robert, L. et al. How other countries use deprivation indices-and why the United States desperately needs one. Health Aff. 35, 1991-1998 (2016).

41. Joint Committee on Vaccination and Immunisation. Joint Committee on Vaccination and Immunisation: Advice on Priority Groups for COVID-19 Vaccination. https://www.gov.uk/government/publications/prioritygroups-for-coronavirus-covid-19-vaccination-advice-from-the- jcvi30-december-2020/joint- committee-on-vaccination- and-immunisationadvice-on-priority- groups-for-covid-19-vaccination-30-december-2020 (January 6, 2021).

42. Acharya, R. \& Porwal, A. A vulnerability index for the management of and response to the COVID-19 epidemic in India: an ecological study. Lancet Glob. Health 8, e1142-e1151 (2020).

43. Paltiel, A. D., Schwartz, J. L., Zheng, A. \& Walensky, R. P. Clinical outcomes of a COVID-19 vaccine: implementation over efficacy. Health Aff. 40, 42-52 (2020).

44. Weintraub, R. L., Subramanian, L., Karlage, A., Ahmad, I \& Rosenberg, J. COVID-19 vaccine to vaccination: why leaders must invest in delivery strategies now. Health Aff. 40, 33-41 (2020).

Publisher's note Springer Nature remains neutral with regard to jurisdictional claims in published maps and institutional affiliations.

(c) The Author(s), under exclusive licence to Springer Nature America, Inc. 2021 


\section{Methods}

We obtained summaries of all jurisdictions' allocation plans published by 8 November 2020, on the CDC's dedicated website ${ }^{2}$. Where a document linked to full guidance, we included it in the analysis and additionally obtained full plans by searching jurisdictions' health department websites through to 30 March 2021 (see description of sequential searches below; archived copies of all retrieved documents are available upon reasonable request). Note that four states (NY, PA, IL and TX) include cities that are themselves CDC jurisdictions (New York City, Philadelphia, Chicago, Houston and San Antonio). For analytic purposes, and in line with the CDC's taxonomy, we captured these cities separately rather than including them in the states they fall within.

Given the rapid pace of updating evolving guidance, we supplemented the initial search with a web browser search. All researchers used the same browser, unlinked to any personal profiles, and searched for the health department name in combination with keywords corresponding to the terms emerging as pertinent from the initial search (see description under Step 3).

Plans and subsequent supplemental documents were analyzed using a nine-item extraction tool conceptualized by H.S., M.A.W. and L.G. and refined in discussions with A.S. and R.W. and eliciting:

1. Whether jurisdictions intended to use a disadvantage index or zip code-based measure (two items);

2. Whether indices were used for prioritizing disadvantaged groups through larger allocations and, if so, what share of what population should be prioritized and to what extent (two items); and whether indices were used to define priority groups for other uses of prioritization, for planning outreach and communication, for planning dispensing sites or for monitoring uptake (1 item each);

3. Whether planners indicate use of the Tiberius software (which might include prioritized allocations to disadvantaged areas via SVI weights) (one item).

Four authors (A.D., E.S., H.W. and N.N.) completed data extraction for all formal allocation plans and supplemental documents; each data source was analyzed in parallel by two analysts. E.S. and H.S. led data entry verification. Any changes in subsequent documents were reviewed collectively (H.S., E.S., H.W., N.N. and A.D.). Differences in data capture were marginal, given the simplicity of the extraction tool, and resolved by consensus.

Data acquisition and extraction. Step 1: Initial data analysis (7-14 November 2020). Data extraction tool was used for all formal jurisdiction summary and full guidance documents.

H.W. and A.D.-Each completed data extraction for half of jurisdictions; E.S.Verified all data entry; A.D., E.S., H.S. and H.W.--Resolved any differences in data capture.

Step 2: Full guidance updates (8-14 December 2020; 21 December 2020-1 January 2021; and 7-19 January 2021). Monitoring for updates to jurisdictions' full guidance documents. Data extraction tool was used for all updated documents.

A.D., E.S., H.W. and N.N.-Each monitored one-quarter of jurisdictions for updates to full guidance documents.

A.D., E.S., H.S., H.W. and N.N.--Resolved any differences in data capture.

Step 3: Supplemental document search (13-19 January 2021; and 20-30 March 2021). An additional search was done to identify supplemental documents on the jurisdictions' health department websites aside from the formal allocation plans that identified use of an index of disadvantage, a reserve system or the Tiberius platform. Google Incognito mode was used to search the following:

1. '[state website url]' covid vaccine Reserve

2. '[state website url' covid vaccine 'Categorized priority system'

3. '[state website url' covid vaccine Tiberius

4. '[state website url' covid vaccine 'vulnerability index'

5. '[state website url]" covid vaccine 'deprivation index'

6. '[state website url]' covid vaccine 'equitable distribution'

For the March 20-30 supplemental document search, an additional search was added as below:

1. '[state health department name]' covid vaccine Reserve

2. '[state health department name]' covid vaccine 'Categorized priority system

3. '[state health department name]' covid vaccine Tiberius

4. '[state health department name]' covid vaccine 'vulnerability index'

5. '[state health department name]' covid vaccine 'deprivation index'

6. '[state health department name]' covid vaccine 'equitable distribution'

7. '[state health department name]' covid vaccine zip

8. '[state website url]' covid vaccine zip

A.D., E.S., H.W. and N.N.-Each completed search as described above for one-quarter of the jurisdictions and completed data extraction for identified documents. A.D., E.S., H.S., H.W. and N.N.-Resolved any differences in data capture.
Step 4: Cross-checking. Finally, A.D., E.S., H.W. and N.N. switched sets of states to verify mentions in the documents found by the other individual. Additionally, searches were completed for the partner's states as described in Step 3. At each of the four overall steps, H.S. and E.S. verified all data extraction for accurate capture.

Supplementary Table 1 provides all central verbatim sections of jurisdictions' uses of disadvantage indices and zip codes. For the full extracted data, see Supplementary Data.

Data on quantifying shares of disadvantaged populations and the impact of statistical measures of disadvantage for adjusting allocations. We sought to classify jurisdictions by their degree of disadvantage. For this part of the analysis, our goal was to classify jurisdictions by the degree of disadvantage or vulnerability of their populations to compare that to their use of a disadvantage index. This analysis matters as, during the period of the analysis (November 2020-March 2021), vaccines were allocated to jurisdictions proportionate to population. If all jurisdictions had the same share of disadvantaged people, these groups would stand an equal chance of getting a vaccine. To the extent that this is not the case, equity issues become the more important: the larger the share of disadvantaged people in a jurisdiction is, the relatively worse chance they stand of receiving a vaccine.

To classify jurisdictions, modifying an earlier analysis that focused on the state, as opposed to jurisdiction level ${ }^{3}$, we started with the 2018 nationwide census tract SVI data, as made available on the CDC's website (https://www.atsdr.cdc.gov/ placeandhealth/svi/data_documentation_download.html). For the four states with city-level jurisdictions within them, we classified census tracts by county, as shown in Supplementary Table 2.

For the remaining 46 states and DC, we counted each census tract as within its state jurisdiction.

The SVI score is a percentile with uniform distribution over all census tracts in the country. A higher SVI means more disadvantage, so an SVI score of 0.75 or higher signifies that the census tract is in the highest quartile of disadvantage relative to a nationwide standard. We flagged each census tract as disadvantaged if its SVI $\geq 0.75$, using the omnibus variable RPL_THEMES for SVI.

We then collapsed the census tracts to the jurisdiction level, summing up the total population of the jurisdiction as well as the population living in high-disadvantage SVI census tracts, using the population variable E_TOTPOP. Finally, for each jurisdiction, we calculated the percent of its total population living in high disadvantage areas, as shown in Extended Data Fig. 1.

As displayed, 20 jurisdictions have more than one-quarter of their population living in areas of high disadvantage, including all five cities and DC. (Alabama's result is $24.538 \%$, just under the $25 \%$ threshold.)

Using the pragmatic threshold of $25 \%$, used in studies by the CDC with similar aims of assessing the impact on particularly disadvantaged populations ${ }^{6}$, half of jurisdictions with a high level of disadvantage are using an index (10/20) as are $56 \%$ of the non-disadvantaged (20/36).

The difference between these proportions is sensitive to the choice of threshold. Extended Data Fig. 2 displays the percent of jurisdictions using an index at a variety of threshold choices.

At the time NASEM recommended setting aside a $10 \%$ national reserve to be allocated to disadvantaged populations as captured under SVI, it was unclear what quantitative impact that this would have in terms of the numbers of doses offered to these communities. To quantify this, we simulated using SVI along a modified version of the index that reduced legal challenges and another index that likewise reduces this risk (the ADI) ${ }^{23}$. Extended Data Fig. 3 shows, on the left-hand side, the consequences of setting aside $10 \%$ at the state level (the more realistic approach; see the example of Tennessee, as noted in the manuscript) of the amount allocated to states based on population and adding this in addition to the share that a state's worse-off quartile, as captured on the respective index, would receive. The right-hand side shows the consequences of doubling this amount to $20 \%$, which can also give a rough idea of what a combined $10 \%$ reserve at the national level and at the state level would mean ${ }^{3}$.

The share of the minority populations that would be offered vaccines under the unadjusted NASEM framework in shown in the gray line. In the initial phase, all indices would offer disadvantaged populations vaccines above their population share, even though, in the case of the unadjusted NASEM framework, the margin is slim and considerably higher on the different indices. Around halfway through phase 1 , using only the state-level $10 \%$ reserve (left-hand side illustration) on all scenarios, the share of offered vaccines drops below the population share, whereas increasing the reserve size to $20 \%$ leads to offers that are consistently above the population share. Note, also, the shares of COVID-related deaths (crude and age-adjusted) of all minority populations collectively that are shown for context on the vertical axis. Furthermore, note that the standardized assumptions made here set aside logistical complexities of implementation, which make it harder, rather than easier, to reach disadvantaged groups, and, for the purpose of illustration, that we (counterfactually) assume that everyone who is offered a vaccine will take one, to err on the side of not overstating our findings.

Reporting Summary. Further information on research design is available in the Nature Research Reporting Summary linked to this article. 


\section{Data availability}

All extracted data are provided in the Supplementary Data. Because more than 300 files were retrieved for the 64 jurisdictions, and a large share comprises considerable numbers of pages, full source data are not included in the Supplementary Data. The documents have, however, been archived and indexed and are freely available upon reasonable request from the authors.

\section{Acknowledgements}

The authors received no specific funding for this work.

\section{Author contributions}

Conceptualization: H.S., M.A.W. and L.G. (refined in discussions with A.S. and R.W.). Data acquisition and extraction: A.D., E.S., H.W. and N.N. Data verification and analysis: A.D., E.S., H.W., N.N. and H.S. Data visualization: A.D., K.M. and E.S. First draft and leading subsequent revisions of the manuscript: H.S. Substantive contributions to manuscript: all authors.

\section{Competing interests}

The authors declare no competing interests.

\section{Additional information}

Extended data is available for this paper at https://doi.org/10.1038/s41591-021-01379-6.

Supplementary information The online version contains supplementary material available at https://doi.org/10.1038/s41591-021-01379-6.

Correspondence and requests for materials should be addressed to H.S.

Peer review information Nature Medicine thanks Govind Persad, Jonathan Wolff and Rachel Gur-Arie for their contribution to the peer review of this work.

Jennifer Sargent was the primary editor on this article and managed its

editorial process and peer review in collaboration with the rest of the editorial team.

Reprints and permissions information is available at www.nature.com/reprints. 
Jurisdiction

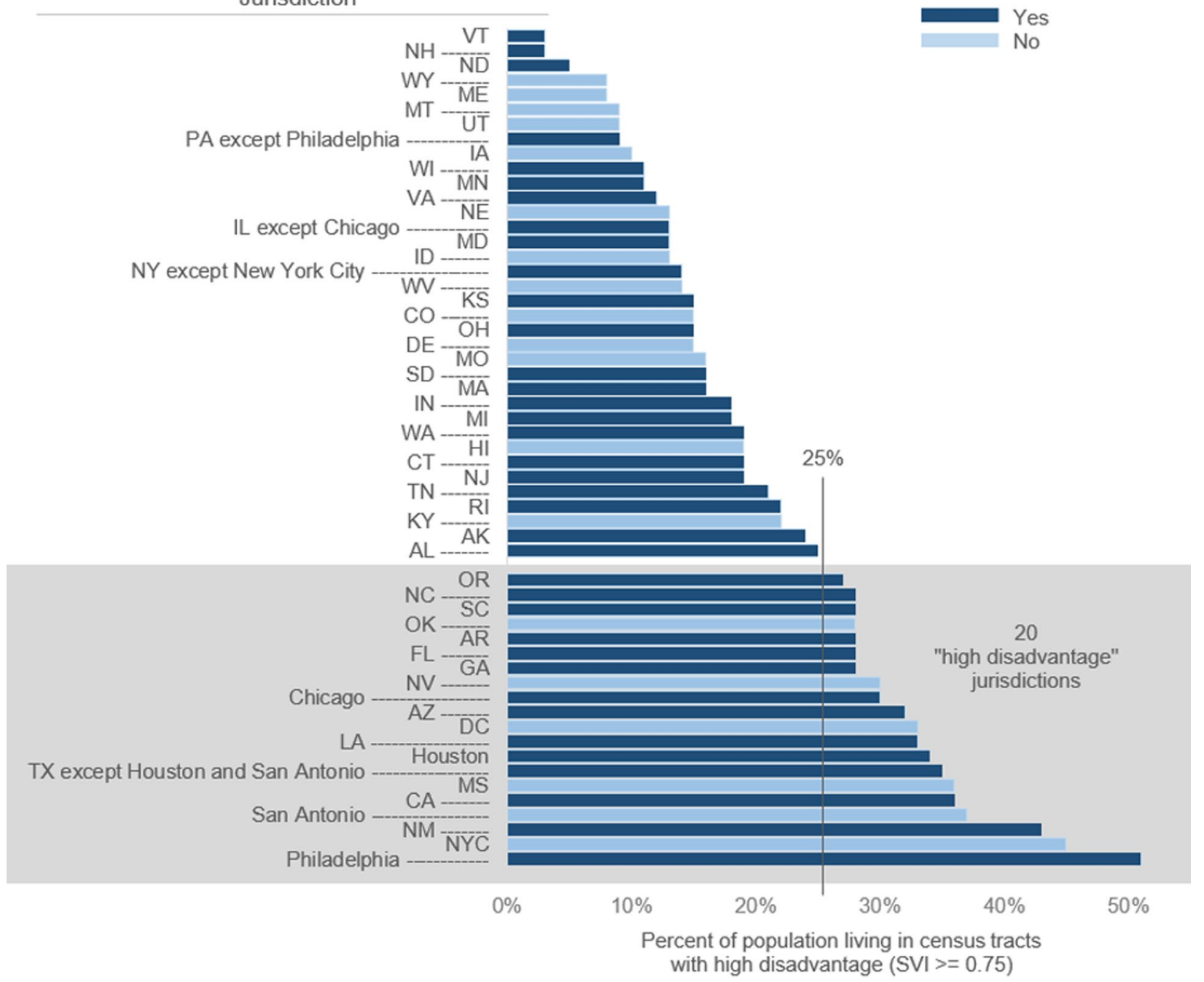

Extended Data Fig. 1 | Percent of population living in high disadvantage census tracts, by use of a disadvantage index. 


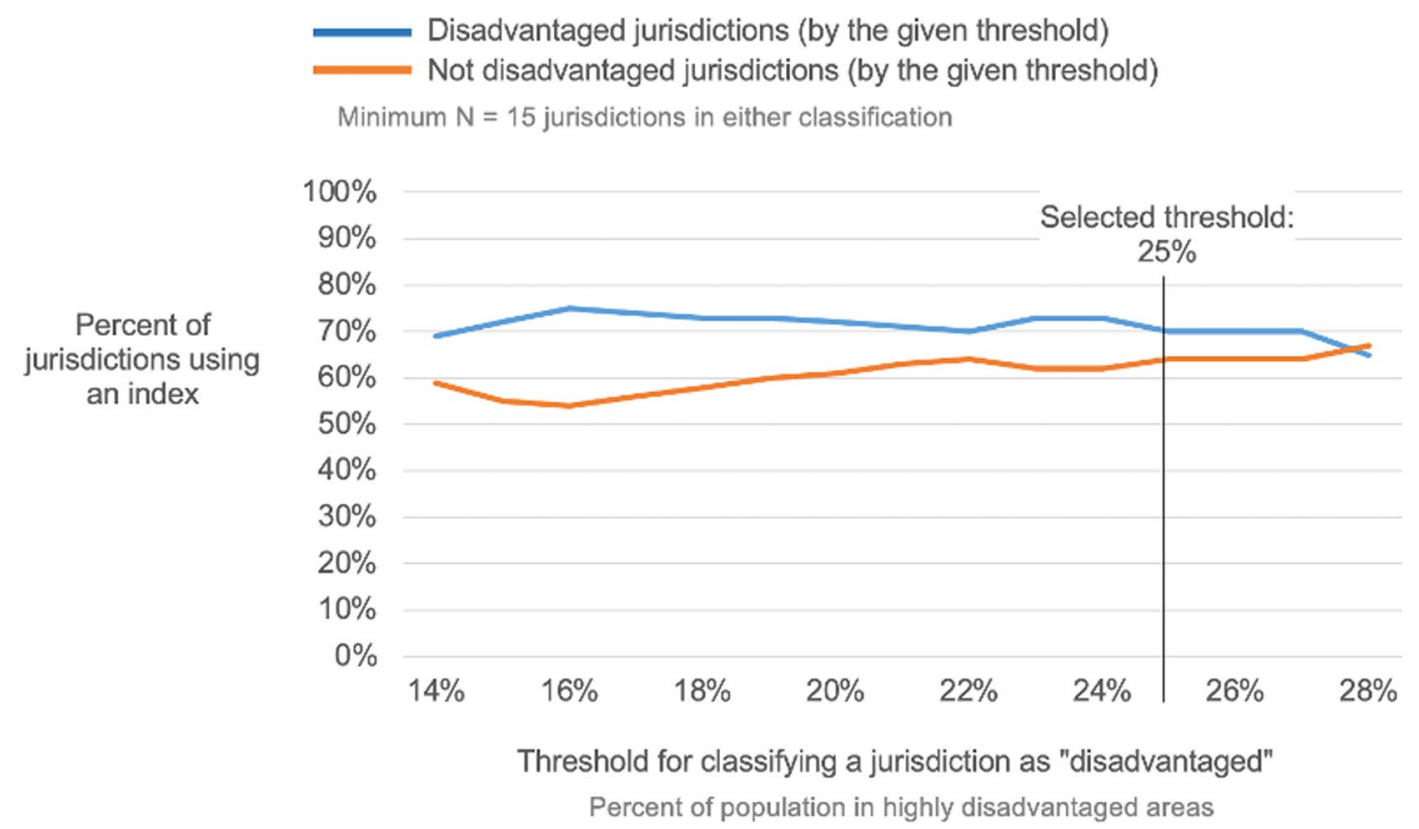

Extended Data Fig. 2 | Percent of jurisdictions using an index, by threshold for classification as a "disadvantaged" jurisdiction. 


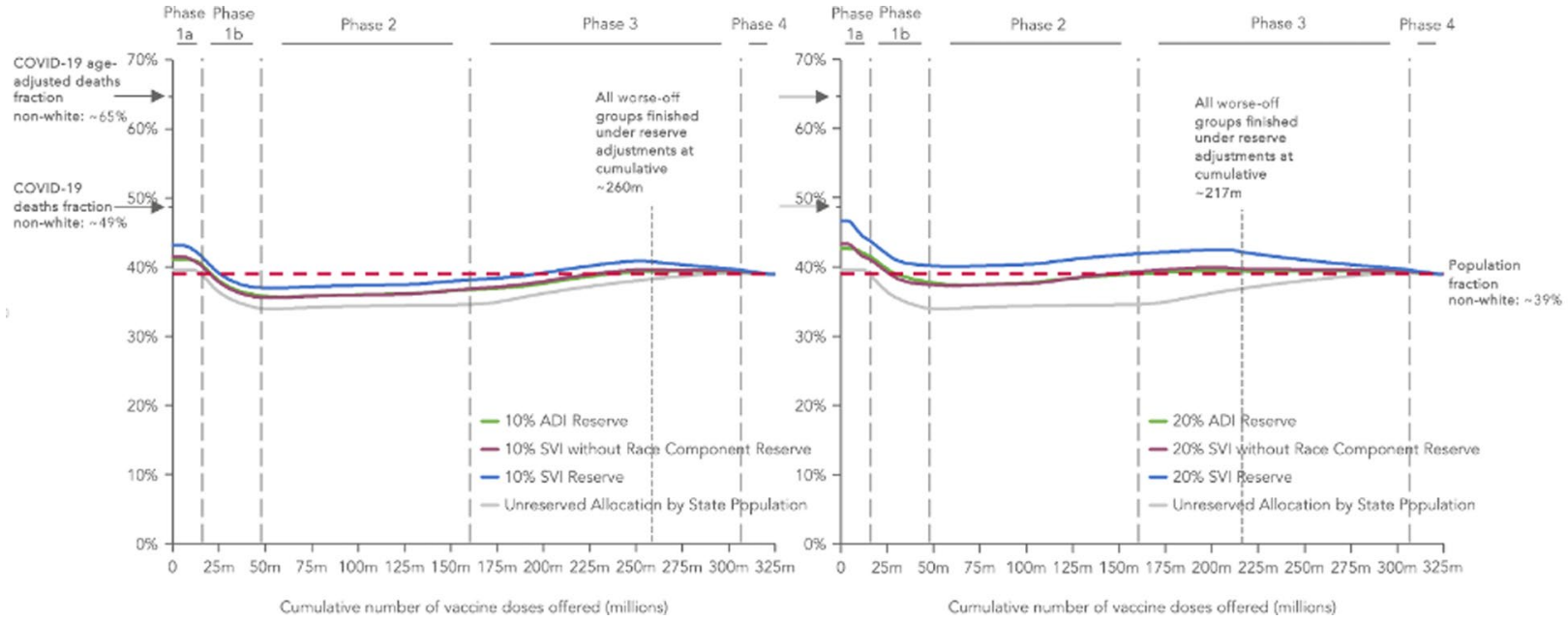

Extended Data Fig. 3 | Proportion of vaccine doses offered to minorities (that is all Hispanic, Black, Asian, Indigenous, mixed-race groups), after and before reserve adjustments for worse-off groups, by cumulative number of vaccine doses offered. Note: Covid-19 death fraction and age-adjusted death fraction are shown as of 09/15/2020. Death rates and age-adjusted death-rates may be inaccurate in the presence of multi-racial groups and racial groups outside of Indigenous, Asian, Black, Latino, white, and Pacific Islander. Non-whites includes all racial groups excluding non-Hispanic white. Phase labels at top of figure represent estimates of approximate NASEM phase populations. All worse-off groups are finished to be served at the end of $323 \mathrm{~m}$ cumulative vaccines when there are no reserve adjustments. Sources: National Academies of Sciences Engineering and Medicine. A Framework for Equitable Allocation of Vaccine for the Novel Coronavirus. Washington, DC: 2020; American Community Survey (ACS) 5 Year; APM Research Lab, Color of Coronavirus. For methods see: Schmidt H, Ünver, Utku, Williams, Michelle; Pathak, Parag; Sönmez, Tayfun; Gostin, Lawrence; What Prioritizing Worse-Off Minority Groups for COVID-19 Vaccines Means Quantitatively: Practical, Legal and Ethical Implications SSRN Working Paper. 2020, available at: https://papers.ssrn.com/sol3/papers.cfm?abstract_id=3716686. 


\section{Reporting Summary}

Nature Research wishes to improve the reproducibility of the work that we publish. This form provides structure for consistency and transparency in reporting. For further information on Nature Research policies, see our Editorial Policies and the Editorial Policy Checklist.

\section{Statistics}

For all statistical analyses, confirm that the following items are present in the figure legend, table legend, main text, or Methods section.

n/a Confirmed

\ $\square$ The exact sample size $(n)$ for each experimental group/condition, given as a discrete number and unit of measurement

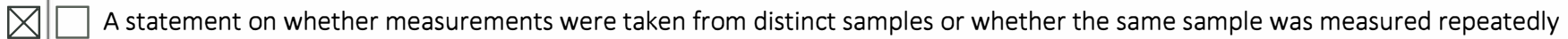

$\square$ The statistical test(s) used AND whether they are one- or two-sided

$\triangle$ Only common tests should be described solely by name; describe more complex techniques in the Methods section.

$\bigotimes \square$ A description of all covariates tested

$\bigotimes \square$ A description of any assumptions or corrections, such as tests of normality and adjustment for multiple comparisons

凶 A full description of the statistical parameters including central tendency (e.g. means) or other basic estimates (e.g. regression coefficient) AND variation (e.g. standard deviation) or associated estimates of uncertainty (e.g. confidence intervals)

Хa For For null hypothesis testing, the test statistic (e.g. $F, t, r)$ with confidence intervals, effect sizes, degrees of freedom and $P$ value noted Give $P$ values as exact values whenever suitable.

\ $\square$ For Bayesian analysis, information on the choice of priors and Markov chain Monte Carlo settings

$\bigotimes \square$ For hierarchical and complex designs, identification of the appropriate level for tests and full reporting of outcomes

$\triangle \square$ Estimates of effect sizes (e.g. Cohen's $d$, Pearson's $r$ ), indicating how they were calculated

Our web collection on statistics for biologists contains articles on many of the points above.

\section{Software and code}

Policy information about availability of computer code

Data collection n/a [Microsoft excel version 16.48 and word tables were used to oorganize data, but no software of any type was used for analytic purposes]

Data analysis a 9 item extraction tool was used, capturing whether index or zip code was used, which types of uses indices were put to, and wheth

For manuscripts utilizing custom algorithms or software that are central to the research but not yet described in published literature, software must be made available to editors and reviewers. We strongly encourage code deposition in a community repository (e.g. GitHub). See the Nature Research guidelines for submitting code \& software for further information.

Data

Policy information about availability of data

All manuscripts must include a data availability statement. This statement should provide the following information, where applicable:

- Accession codes, unique identifiers, or web links for publicly available datasets

- A list of figures that have associated raw data

- A description of any restrictions on data availability

All extracted data from jurisdiction policy documents is presented at Annex A. Full archived copies of all retrieved jurisdiction policy documents are available from the corresponding author 
Please select the one below that is the best fit for your research. If you are not sure, read the appropriate sections before making your selection.

Life sciences $\quad \bigotimes$ Behavioural \& social sciences $\square$ Ecological, evolutionary \& environmental sciences

For a reference copy of the document with all sections, see nature.com/documents/nr-reporting-summary-flat.pdf

\section{Behavioural \& social sciences study design}

All studies must disclose on these points even when the disclosure is negative.

Study description

Research sample

Sampling strategy

Data collection

Timing

Data exclusions

Non-participation

Randomization
Qualititative analysis of jurisdiction's use of disadvantage indices. Quantitative analysis to the extent that we provide counts of jurisdiction that use indices, as well as percentages by use and type of use.

All 64 US jurisdictions, as defined by the $\mathrm{CDC}$ (the sample constitutes the exclusive and exhaustive universe of administratiive units that allocate vaccines in the US)

We analyzed documents from the entire universe of jurisdictions--no sampling was performed

We obtained summaries of all jurisdictions' allocation plans published by November 8 on the CDC's dedicated website.1 Where a document linked to full guidance, we included it in the analysis, and additionally obtained full plans by searching jurisdictions' health department websites through to March 30, 2021 (all search periods: November 7-14, 2020; December 8-14; December 21-January 1, 2021; January 7-19; and March 20-30) Plans and subsequent supplemental documents were analyzed using a nine-item extraction tool for organizing data as the analytic instrument. Four authors ( $A D, E S, H W, N N)$ completed data extraction for all formal allocation plans and supplemental documents; each data source was analyzed in parallel by two analysts. ES and HS led data entry verification. Any changes in subsequent documents were reviewed collectively (HS, ES, HW, NN and AD). Differences in data capture were marginal, given the simplicity of the extraction tool, and resolved by consensus

no data that were excluded from the analysis

since this was a descriptive study based on documents retrieved by applying purposive keywords and since the study did not involve participants, non-participation is not applicable.

since this was a descriptive study based on documents retrieved by applying purposive keywords, randomization was not applicable

\section{Reporting for specific materials, systems and methods}

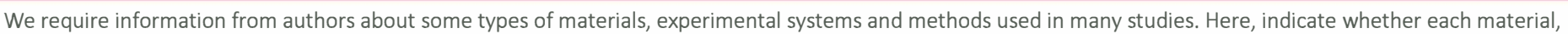

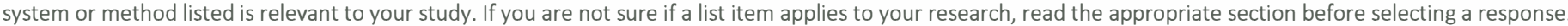

Materials \& experimental systems

\begin{tabular}{l} 
Methods \\
\hline n/a $\mid$ Involved in the study \\
$\square$ \\
$\square$ ChIP-seq \\
$\square$ \\
$\square$ Flow cytometry \\
$\square$ MRI-based neuroimaging
\end{tabular}

$\mathrm{n} / \mathrm{a}$ Involved in the study

$\triangle \square$ Antibodies

\ $\square$ Eukaryotic cell lines

$\bigotimes \square$ Palaeontology and archaeology

$\bigotimes \square$ MRI-based neuroimaging

$\triangle \square$ Animals and other organisms

\ $\square$ Human research participants

\ $\square$ Clinical data

$\bigotimes \square$ Dual use research of concern 